\title{
Editorial
}

\section{Malaria: an eradicable threat?}

\author{
Alfonso J. Rodriguez-Morales. \\ J Infect Developing Countries 2008; 2(1):1-2. \\ Received 8 January 2008 - Accepted 11 January 2008. \\ Copyright (? 2008 Rodriguez-Morales. This is an open access article distributed under the Creative Commons Attribution License, which permits \\ unrestricted use, distribution, and reproduction in any medium, provided the original work is properly cited.
}

The global burden of malaria stills represents a significant threat in the developing world. More than 100 countries and territories have areas at risk of malaria transmission, with around 3.2 billion people living in these areas [1]. An estimated 350 to 500 million clinical malaria episodes occur annually; most of these are caused by infection with Plasmodium falciparum and $P$. vivax. The first causes more than 1 million deaths each year, also contributing indirectly to many additional deaths, mainly in young children, through synergy with other infections and illnesses [1]. Unfortunately, approximately $60 \%$ of the world's cases of malaria, including close to $75 \%$ of the global falciparum malaria cases, occur in Sub-Saharian Africa [1]. Furthermore, over $80 \%$ of worldwide malaria deaths occur in this region [1]. This terrible scenario is the target of physicians and multidisciplinary researchers worldwide who aim to halve the burden of this humankind threat by 2010 (Global Partnership to Roll Back Malaria, World Health Organization, Abuja declaration. Geneva: WHO. Available online: http://www.rbm. who.int/docs/abuja_declaration_final.htm).

Recently, the Gates Foundation called on global leaders to embrace "an audacious goal-to reach a day when no human being has malaria, and no mosquito on earth is carrying it" (Bill and Melinda Gates Foundation, Bill and Melinda Gates Call for New Global Commitment to Chart a Course for Malaria Eradication. October 17, 2007. Available online: http://www.gatesfoundation.org/ GlobalHealth/Pri_Diseases/Malaria/Announcement s/Announce-071007.htm). Melinda Gates stated: "Advances in science and medicine, promising research, and the rising concern of people around the world represent an historic opportunity not just to treat malaria or to control it -but to chart a long-term course to eradicate it,"
(http://www.gatesfoundation.org/GlobalHealth/Pri Diseases/Malaria/Announcements/Announce-071 007.htm). Tadataka Yamada, president of the Gates Foundation's Global Health Program, made a case for eradication during the American Society for Tropical Medicine and Hygiene Annual meeting in December 2007 [2]. He underlined reasons that it was possible, even necessary, to consider malaria eradicable in the near future. Given the failure of an earlier eradication program, this new message gives reason for optimism, but the question remains: is malaria truly an eradicable disease? Based on experiences from the recent past, many public health physicians would say no, but perhaps current knowledge is insufficient to answer the question. However, even realists would concede that malaria could be a controllable disease.

Although the Global Fund to Fight AIDS, Tuberculosis, and Malaria (GFATM), as well as the World Bank's Malaria Booster Program and the US President's Malaria Initiative are bringing new energy and resources to the global effort to control malaria [1] , this is not yet enough. Together, these initiatives have committed $\$ 3.6$ billion to malaria control, but in spite of this, malaria morbidity and mortality is significantly increasing in many countries in Africa, as well also in Asia and Latin America, perhaps reflecting that even this impressive economic commitment is not enough to decrease malaria's impact on health and human life in developing countries. To help build on progress against malaria, Mr. and Mrs. Gates called on US presidential candidates to commit to supporting the President's Malaria Initiative, a \$1.2 billion effort launched by President Bush in 2005 (http://www.gatesfoundation.org/GlobalHealth/Pri Diseases/Malaria/Announcements/Announce-071 007.htm). Mr. Gates said, "If you win this office, 
you will inherit a record commitment to fighting malaria. The world needs you to sustain it and enhance it. Malaria will never be eradicated without the full support of the President of the United States." It also goes without saying that malaria will never be eradicated without the full support of the presidents of both donor and affected countries.

As governments, donors and health systems delay implementation of life-saving interventions such as insecticide treated bed-nets, vector control, improved diagnostics and effective treatments, the disease and its landscape continue to change, bringing new complications. Evidence continues to accumulate to support the view that adults infected with HIV, in addition to children under 5 years of age and pregnant women, should be targeted for malaria prevention and treatment. Malaria contributes synergistically with HIV/AIDS to morbidity and mortality in areas where both infections are highly prevalent, such as in subSaharian Africa. In addition to providing immediate health benefits, prevention and treatment of malaria may lessen transient increases in HIV viral load during malaria episodes and thus help limit the progression and transmission of HIV [1]. Moreover, recently a new parasite species, at least well confirmed in Asia, has been added to the list of etiological agents of human malaria, in addition to $P$. falciparum, $P$. vivax, $P$. malariae and $P$. ovale, the so-called fifth human malaria parasite, P. knowlesi $[3,4]$.

Although much progress has been made in research and in the control of the malaria scourge since 1997 when the Multilateral Initiative on Malaria (MIM) began as an international alliance of organizations and individuals concerned about the state of malaria research [5], there is still a long way to go. In 1998, Roll Back Malaria, now the Global Malaria Programme, and the Roll Back Malaria (RBM) Partnership began at the World Health Organization (WHO). Increasingly, research and control programs have focused on Africa where the overwhelming malaria burden occurs. One of the greatest benefits of these and other malaria initiatives has been the application of research findings to patient management and disease prevention. The development of sustainable malaria research and control programs, particularly in Africa, remains a top priority of many new efforts to combat malaria while raising international public awareness and attracting resources to combat this scourge [5]. As has been mentioned, among these many new partners joining the fight against malaria, the Bill and Melinda Gates Foundation, the Global Fund Against HIV/AIDS, Tuberculosis, and Malaria and the U.S. President's Malaria Initiative have contributed funds, expertise and leadership.

The Gates Foundation will begin to call for innovative proposals to overcome existing roadblocks to eradication later this year. Bill Gates recently stated, "Both private industry and public research institutions must continue to invest in new tools in order to make malaria eradication possible" (http://www.gatesfoundation.org/Global Health/Pri_Diseases/Malaria/Announcements/Ann ounce-071 007.htm), then sustained in time. If this happens the initial question raised in this editorial could be answered "Yes."

\section{References}

1. Global Partnership to Roll Back Malaria, World Health Organization, and UNICEF (2005). World malaria report 2005. Geneva: World Health Organization: UNICEF. xxxi, 1-294.

2. Yamada T. (2007). Perspectives in Global Heath. Plenary Session I, American Society of Tropical Medicine and Hygiene 56th Annual Meeting, November 4-8, 2007, Philadelphia, Pennsylvania. USA. Final Program. Am J Trop Med Hyg;77 (5 Suppl):45.

3. Cox-Singh J, et al. (2008) Plasmodium knowlesi Malaria in Humans Is Widely Distributed and Potentially Life Threatening. Clin Infect Dis;46 (2):165-171.

4. White NJ (2008) Editorial Commentary: Plasmodium knowlesi: The Fifth Human Malaria Parasite. Clin Infect Dis;46(2):172-173.

5. Breman JG, Alilio MS, White NJ (2007) Defining and Defeating the Intolerable Burden of Malaria III. Progress and Perspectives. Am J Trop Med Hyg;77 (6 Suppl):i.

Corresponding Author: Alfonso J. Rodriguez-Morales, Experimental Institute José Witremundo Torrealba (former Center for Parasitological Research JWT), Universidad de Los Andes, Trujillo, and Immunoparasitology Section, Tropical Medicine Institute, Universidad Central de Venezuela, Caracas, Venezuela. E-mail: alfonsorm@ula.ve

Conflict of interest: No conflict of interest is declared. 